\title{
LITERATURWISSENSCHAFT
}

http://dx.doi.org/10.18778/2196-8403.2016.08

JOANNA FIRAZA

\section{Triumphe des Heidentums: Bildende Kunst in Frank Wedekinds Franziska}

Franka Wedekinda ,myślenie obrazami‘ szczególnie dobrze prześledzić można w jego późnym dramacie Franziska (1911). Tendencja wizualizacyjna przejawia się tu z jednej strony w zdjęciowej choreografii scenicznej, z drugiej w licznych odniesieniach do historii sztuki, poprzez które autor zabiera głos we współczesnym mu dyskursie. Cytaty obejmują szeroki okres od renesansu, poprzez prerafaelitów aż po sztukę współczesną, co otwiera bogate archiwum obrazów z figuracjami Fausta, Heleny, Mignon, Ewy, Maryi i innych. Cytaty optyczne pełnią funkcje ironicznej korekty tradycji chrześcijańskiej, dzięki czemu autor wpisuje się w sensualistyczne dziedzictwo antyku.

Frank Wedekinds ,Denken in Bildern“ lässt sich im späten Stück Franziska (1911) besonders gut verfolgen. Zum einen manifestiert sich die Visualisierungstendenz in der tableauartigen Szenen-Choreographie, zum anderen in zahlreichen Bildzitaten, mit denen der Autor zum zeitgenössischen Kunstdiskurs Stellung bezieht. Die kunstgeschichtlichen Bezüge umfassen die breite Palette von der Renaissance, über die Präraffaeliten bis zur Kunst der 10er Jahre des 20. Jahrhunderts, womit ein reichhaltiges Bildarchiv mit u.a. Faust-, Helena-, Mignon-, Eva- und Maria-Figurationen eröffnet wird. Die visuellen Zitate fungieren als ein ironisches Korrektiv der christlichen Tradition, womit sich Wedekind als Sensualist in der Nachfolge der Antike profiliert.

Frank Wedekind's visual thinking can be studied particularly clearly in his late drama Franziska (1911). The tendency to visualize is on the one hand visible in the tableau-style choreography and on the other - in numerous references to art history, through which the author takes a position in contemporary art discourse. The references range from Renaissance through Pre-Raphaelite painting to contemporary art, opening up a wide archive of images, among them the figures of Faust, Helena, Mignon, Eve, 
Saint Mary and others. These "optical allusions" form an ironic corrective to the Christian tradition, and allow the author to inscribe his work among the 'sensualists' in the heritage of antiquity.

Frank Wedekinds Kunstverständnis und die Referenz der Kunst-Zitate auf die Ausgestaltung und -bedeutung seiner Dramen ist noch weitgehend unerforscht. Als erster wies Dieter Kafitz auf diesen Mangel hin, ${ }^{1}$ der anhand von Wedekinds Briefen und Tagebucheintragungen auf dessen Aufgeschlossenheit gegenüber den Klassikern der Malerei der Renaissance, besonders der Tizians, sowie gegenüber neueren Kunstbestrebungen aufmerksam machte (vgl. KAFITZ 2001:269). Die Vorliebe der Moderne für die Renaissance ist bekannt, Wedekind stellt hier keine Ausnahme dar. Zudem war die Kunststadt München nicht weniger als Paris, wo Wedekind sich in den 1890er Jahren aufhielt, dem Interesse an der bildenden Kunst durchaus förderlich. ${ }^{2}$ In einer Notiz aus dem Jahr 1889 ist zu lesen: „Vor Tisch gehe ich in die Neue Pinakothek, wo mich vor allem die Maria von Bonveret fesselt, außerdem [...] Böcklins Spiel der Wellen. Vollkommen kalt lassen mich die Pilotyschen Bilder." (Notiz Frank Wedekinds v. 17.07.1889, HAY 1986:92). Das Schaffen des Schweizer Malers Arnold Böcklin (1827-1901), dem Wedekind zuerst 1884/85 begegnet, konnte er auch in der Münchner Schack-Galerie, der Privatkollektion süddeutscher Malerei des mittleren 19. Jahrhunderts betrachten (vgl. AUSTERMÜHL / KIESER / VINÇON 2000:565). ${ }^{3}$ Der Stifter der Galerie Adolf Friedrich von Schack

1 Die zahlreichen Kunstzitate in Frühlingserwachen versteht Dieter Kafitz nicht als Illustrationen, sondern sieht sie in ein dichtes Verweisungsnetz der Dramenhandlung verwoben. Ihm zufolge stehen sie in engem Zusammenhang mit dem Sexualitätsdiskurs im Stück (vgl. KAFITZ 2001:263).

„Von seinen Bildern [Mihály Munkácsy (1844-1900), einer der bedeutendsten ungarischen Maler, seit 1872 in Paris lebend] kennt er [Muth] so gut wie nichts. Ich mache ihn auf die bedeutendsten aufmerksam. Er fragt mich, ob es mich interessieren würde, Munkácsys Atelier zu sehen." Notiz Frank Wedekinds v. 3.12.1892. (HAY 1986:224).

Adolf Friedrich von Schack hatte früh Anselm Feuerbach, Arnold Böcklin und Moritz von Schwind durch Ankäufe gefördert - in der Sammlung befinden sich zudem Bilder von Franz von Lenbach, Carl Spitzweg, Bonaventura Genelli u. a. 2009 wurde die Schack-Galerie zum 100. Geburtstag in "Sammlung Schack“ umbenannt. 
(1815-1894), Diplomat, Schriftsteller und Kunstkenner, ließ aber auch venezianische Renaissancemaler wie Bellini, Tintoretto oder Tizian kopieren. ${ }^{4}$ Die Tagebücher bezeugen zudem Wedekinds Interesse an den Schriften zur Kunsttheorie. So schrieb er 1905 an Walter Rathenau: „Ihre Aufsätze über Malerei habe ich mit größtem Genuß gelesen. [...] Ich freue mich sehr darauf, mit Ihnen darüber sprechen zu können, wenn Ihre Zeit Ihnen das in nächster Woche gestattet" (STRICH 1924a:151). ${ }^{5}$

Mit dem Hinweis auf Wedekinds Sensibilität gegenüber den bildenden Künsten schlägt Dieter Kafitz eine neue Perspektivierung seiner Texte vor. Er stellt eine ,Dominanz des Visuellen gegenüber dem Dialogischen “ - eine Art ,Visualisierung der Gattung' fest (vgl. KAFITZ 2006:37-39). ${ }^{6}$ Dies manifestiert sich in der Tendenz zu tableauartigen Körperbildern und der übergeordneten Rolle des Bewegungsspiels. Dabei war es Adorno, der als erster einen ähnlichen Ansatz verfolgte, indem er bereits 1932 bei Wedekind ein ,Denken in Bildern' beobachtete (vgl. ADORNO 1974:632) und damit in erster Linie das Tableau als literarisches Stilmittel des Dramatikers meinte. Es bietet sich zudem an, in diesem Kontext Siegrid Dreiseitels Studie von 2000 zu nennen, in der sie auf die Verwandtschaft vieler Werke Wedekinds mit Fotographie und Film hinweist (vgl. DreISEITEL 2000:20). Mit Blick auf die These von der, Visualisierungstendenz' soll im Folgenden das Stück Franziska von 1911 (WEDEKIND 1990a:639-742) betrachtet werden, worin die Rückwirkung des Kunstinteresses des Autors und seiner plastischen Vorstellungskraft sich mehrfach durch Kunstzitate sowie durch Szenen-Choreographie ${ }^{7}$ andeutet.

4 Tizian wurde z. B. 1868 von Lenbach kopiert. Dies tat Schack ,nicht als naiver Sammler, sondern zu Studien, weshalb Schäden und Schmutz genau abgemalt wurden."

5 Mit den Aufsätzen sind Rathenaus Texte zum gegenwärtigen Kunstdiskurs gemeint: Insbesondere die Schrift Von neuzeitlicher Malkunst, sowie die Aphorismen Ungeschriebene Blätter (vgl. REINHART 1905:11-25). Vgl. auch HEIMBÖCKEL 1996:142-144.

6 Mit der Entdeckung des Körpers als eines alternativen Mediums gegenüber der diskursiven Sprache beteiligt er sich, Kafitz zufolge, an der Retheatralisierung des zeitgenössischen Dramas (vgl. KAFITZ 2006:37-39).

7 Eine Sonderstellung gebührt in dieser Hinsicht dem Stück Oaha (1909), worin die Auseinandersetzung mit der Zeitschrift Simplizissimus mit der satirischen Form 
Franziska setzt den Schlusspunkt hinter Wedekinds langjährige Auseinandersetzung mit der Faust-Idee (vgl. IRMER 1975:206-214), die sich hier im Konzept eines weiblichen Fausts, einer ,Faustine“ realisiert. ${ }^{8}$ Dem Wunsch Franziskas gemäß vollzieht sich im zweiten Akt der Rollentausch: die Verwandlung zu einem Mann und die Erprobung der Existenz unter anderen sozialen Bedingungen. Wie im Drama Musik wird die Titelfigur zur Sängerin ausgebildet, um - schon als Franz - die Welt im Rahmen von Gastauftritten zu bereisen. Auffallend ist bereits die nur lose Verknüpfung von neun ,Bildern', die so den Charakter von heterogenen, die Metamorphosen der Hauptfigur vorführenden Genrebildern bekommen. Mit der bevorstehenden Wandlung Franziskas kündigt sich der Eintritt in die Welt der Phantasie an. Wedekind spielt dabei direkt auf ein großes Bildarchiv an: es hängt in erster Linie mit dem Faust-Stoff und dessen Bearbeitungen zusammen - vom Volksbuch über Heines Ballettszenario Der Doktor Faust, Goethes Faust I und $I I^{9}$ bis hin zu Offenbachs Orpheus in der Unterwelt. ${ }^{10}$ Den Komplex ergänzt das Bildarchiv der Mignon-Figur: Dem Text ist ein Motto aus Goethes Venezianischen Epigrammen (1790) vorangestellt: „Wende die Füßchen zum Himmel nur ohne

des Stücks - einer Satire der Satire derart verfließt, dass Choreographien bestimmter Szenen das Idiom der für die Zeitschrift typischen Jugendstilkarikatur bekommen.

Die Bezeichnung , weiblicher Faust' bzw. ,Faustine' geht ursprünglich auf Wedekinds eigene Worte zurück: In einer Aussage v. 8.05.1911 heißt es, er plane einen „weiblichen Faust, Faustine“ (KUTSCHER 1931:114). Vgl. auch MARTIN (2001:86f.) und KUTSCHER (1964:296). So heißt es auch in Wedekinds Einleitung zu einer Lesung aus Franziska in Berlin: ,[I]ch habe nämlich einen weiblichen Faust geschrieben“ (zit. nach MARTIN 2001:81-82). AUSTERMÜHL (1989:343-420) weist aber bereits auf die frühe Lektüre von Heines Buch der Lieder und Der Doktor Faust hin (vgl. auch DREISEITEL 2000:29).

AUSTERMÜHL (vgl. 2003:16-19) analysiert die von Wedekind aufgegriffene und umgedeutete Idee des Faustischen: Im Gegensatz zu Historia von Dr. Johann Fausten und Goethes Faust rezipiere Wedekind die Antike unter Umgehung der Klassik und verfolge eine ,anthropozentrische und antimetaphysische Ausrichtung“ (AUSTERMÜHL 2003:19).

10 „Zwanzigtausend Mark Konventionalstrafe sind hin, / Wenn ich in London den Orpheus nicht singe.“(WEDEKIND 1990a:667). 
Sorge! / Wir strecken / Arme betend empor; aber nicht schuldlos, wie du. "11 Dieser klassische Rahmen wird durch eine Vorstellung gesprengt, die dem Stück einen sehr modernen Stempel aufdrückt: Veit Kunz, die zweite Hauptfigur und der Prototyp Mephistos, informiert Franziska über, vier Bedingungen des Kunstgesangs': $: 12$

Erstens gähnende Rachenstellung [...,] [z]weitens bewegliche Ohren [...,] [d]rittens im Kopf ein gleichschenkliges Dreieck, bestehend aus Mundöffnung, Nasenwurzel und weichem Gaumen [...]. Viertens dürfen Sie beileibe nicht glauben, Sie hätten die Nase mitten im Gesicht. Sie müssen felsenfest davon überzeugt sein, dass sich ihr Mund oberhalb der Nase befindet. (WEDEKIND 1990a:653)

Aus den ,Bedingungen“ ergibt sich ein fragmentiertes und enthumanisiertes Bild des Kopfes, das an einen diszipliniert-fitten, von der Natur entfremdeten Körper denken lässt. Andererseits verweist der spielerische Gestus des Stücks auf eine ironische Polemik gegen eine neue Auffassung vom menschlichen Körper, die sich in der modernen Malerei manifestiert. Der organische Fokus der Bedingungen und ihre antimimetische Tendenz im Rahmen von Wedekinds ,Denken in Bildern “ - der Gestaltung und Verteilung der Körper im Theaterraum - weist gewisse Affinitäten mit dem geometrischen Denken des Kubismus auf. Bei der damals neuen Kunstrichtung handelte es sich um die „Reduzierung der menschlichen Anatomie auf geometrische Rhomben und Dreiecke sowie [um den] Verzicht auf normale anatomische Proportionen“ (FRY 1966:14). ${ }^{13} \mathrm{Zu}$ den

11 Wedekind erklärt das Motto im Berliner Börsen-Courier 415 v. 5.09.1913 in einem offenen Brief an Max Reinhardt mit dem Wunsch, das Mignon-Thema zu erweitern (zit. nach AUSTERMÜHL / KIESER / VINÇON 2009:1033). Vgl. auch Wedekinds Tagebucheintrag v. 7.08.1889 (München): „Abends auf dem HBK denke ich fortwährend des Goetheschen Distichons: Wende die Füße zum Himmel etc." und die Notiz v. 8.08.1889: „Das Distichon verlässt mich nicht mehr.“ (HAY 1986:107f.)

Denkbar ist hier ein Bezug auf die Stimmlehre des Physiognomikers Ottmar Rutz (1881-1952). (AUSTERMÜHL / KIESER / VINÇON 2009:1056).

13 „Wo wir glauben, eine weibliche Figur entdecken zu können, weil wir im Katalog gelesen haben: Femme nue, hat der Künstler allein die geometrischen Harmonien im Auge gehabt, in denen sich ihm die gesamte Natur darstellt. [...] Niemand befasst sich weniger mit Psychologie als er [...].“ So Charles Morice über George Braque und dessen Ausstellung in der Kahnweiler-Galerie 1908 in Mercure de France von 1908 (zit. nach FRY 1966:59). Daniel-Henry Kahnweiler war ein deutscher Kunsthändler und Galerist in Paris, den Picasso 1910 porträtierte. „Ich vermöchte es nicht, eine Frau in all ihrer natürlichen Schönheit darzustellen. [...] Ich muß deshalb eine neue Art von Schönheit schaffen, die Schönheit, die sich mir als Volumen, Linie, Masse, Gewicht zeigt. [...] Ich möchte das Absolute der Frau, 
weiter tradierten formalen Prinzipien der Ästhetik des Kubismus gehört u.a. die Idee des ,tableau-objet', die Flächigkeit der Bildebene, die nicht illusionistischen Wechselbeziehungen zwischen den Bildebenen sowie die Collage (vgl. FRY 1966:49). Der Kubismus entwickelte sich zwischen 1907 und den mittleren und späten zwanziger Jahren, allerdings blieb er bis um 1912 ein ausschließlich Pariser Phänomen. Erst große Salons - der Salon des Indépendants im Frühjahr 1911 und der Salon d'Automne 1911 sorgten für größere Öffentlichkeit, so dass die zunächst ignorierten Maler plötzlich zu einer ,Offenbarung' (vgl. GlEIZES 1928:10f.) wurden und der Begriff ,Kubismus' sich durchsetzte. Zum Zeitpunkt der Entstehung von Wedekinds Stück 1911 hatte Pablo Picasso das wegbereitende Bild des Kubismus, Les Demoiselles d'Avignon (1907), jedoch bereits geschaffen. ${ }^{14}$ Das Revolutionäre an diesem Bild war die Tatsache, dass sich der Maler hier von ,zwei Hauptmerkmalen der europäischen Malerei seit der Renaissance losmachte: der klassischen Norm für die menschliche Gestalt und dem Raum-Illusionismus der Zentralperspektive“ (FRY 1966:14). Picasso schaffte es z. B., die Profilansicht und die en-faceAnsicht eines Gesichts zu verbinden, denn ,[e]in Kopf ist eine Sache aus Augen, Nase und Mund, die verteilt werden können, wie immer man will. Der Kopf bleibt ein Kopf.“ (FRY 1966:47) ${ }^{15}$ Picassos Gemälde stellt eine Bordellszene mit fünf Frauen dar. Signifikant ist, dass Wedekind seine ,Hexenküche“ - die ,Weinstube Clara' - ausgerechnet als ein Berliner Etablissement gestaltet, ${ }^{16}$ um dort eine Parodie des Kunstdiskurses zu verorten. Die Weinstubenszene hat eine filmische Dramaturgie: Der Fokus verlagert sich mehrmals von einem der drei Tische zu einem anderen. Veit Kunz ist es, der den Charakter dieser Gesellschaft auf den Punkt bringt: „Schriftsteller und Dirnen! Prolet und Baron! / Hier wird der Verzweifeltste munter. / Uns alle verschwägert ein kindlicher Ton. / Mild lächelt die fleischliche Prostitution / Auf die des Geistes hinunter!“ (WEDEKIND 1990a:657) Ein Beispiel für die ,Prostitution des Geis-

nicht nur ihre äußerliche Erscheinung enthüllen." So lautet Georges Braques Aussage, die auch als erste registrierte Äußerung eines Kubisten über seine Kunst gilt (zit. nach FRY 1966:60).

$14 \mathrm{Zu}$ wegweisenden Künstlern für Picasso werden zu diesem Zeitpunkt u.a. El Greco, Gauguin und Cézanne; auch die afrikanische Plastik ist für sein Schaffen prägend.

15 Picassos Bemerkung von vor 1914 gegenüber Leo Stein. Stein, Leo (1947): Appreciation. Painting, Poetry and Prose. New York: 177 (zit. nach: FrY 1966:47).

16 „Wie weit ist's mit der Hurenrepublik, / Dem Hierodulenstaat.“ (WEDEKIND 1990a:661) 
tes' liefert das dem Publikum nur teilweise zugängliche Gespräch dreier Herren am rechten Tisch: Es fällt der Name [Max] Klingers, vor dessen Heiland einer der Herren Begeisterung empfunden habe. (vgl. WEDEKIND 1990a:656) Die Unterhaltung wird doppelt ironisiert: durch die profane Verortung und die vulgäre, dreimal slapstickartig wiederkehrende Phrase „Halt’s Maul, alte Sau!“ (WEDEKIND 1990a:657, 661, 666). Das kritische Bild des gegenwärtigen Kunstdiskurses kann aber nicht darüber hinwegtäuschen, dass der zitierte Künstlername hier einen Signalwert besitzt und mit der Botschaft des Textes eng verbunden ist.

Der deutsche Maler, Bildhauer und Graphiker Max Klinger (1857-1920) wurde der deutschen Öffentlichkeit durch einen Aufsatz des dänischen Kritikers Georg Brandes 1882 vermittelt. ${ }^{17}$ Wedekind dürfte Klinger 1897 persönlich kennen gelernt haben, als er einem Vortrag der Literarischen Gesellschaft beiwohnte, wo auch Klinger als deren Mitglied anwesend war. Belegt sind zudem zwei Besuche in Klingers Atelier in Leipzig 1900 und 1913 sowie der Erwerb von Klingers Radierzyklus Eine Liebe im Jahr 1907 (AUSTERMÜHL / KIESER / VINÇON 2009:1045). Mit dem im Text erwähnten Titel Heiland könnte die Radierung Der Tod als Heiland aus dem Zyklus Vom Tode I (1889) gemeint sein, denkbar ist aber auch eine Anspielung auf eine von zwei monumentalen, großformatigen Christus-Darstellungen, zugleich Klingers vieldiskutierte Hauptwerke: Die Kreuzigung Christi (1888-1891) oder Christus im Olymp (1883-1897). Zwei Momente könnten dafür sprechen. Zum einen liegt eine gewisse formale Verwandtschaft vor: Klingers Werke sind nämlich gattungsübergreifend, indem sie Malerei, Bildhauerei, Musik und Literatur zu verbinden suchen. Vor allem aber ist es die Grundidee, die diese beiden Gemälde mit Wedekinds Stück Franziska verbindet, und zwar die Vereinigung von Antike und Christentum. Das erste der beiden Bilder stellt zentral Christus und die vor ihm kniende Psyche in der Geste der Handreichung dar. Überblickt man die zahlreichen Vorbilder - mehrere Varianten von Noli me tangere mit der Figuration Maria Magdalenas, Eva-Figurationen, Christus und die Ehebrecherin - ist die Szene bei Klinger als Verführung zum Ehebruch zu deuten: „Der Heiland bekehrt hier niemanden, sondern nimmt sich eine Braut. ${ }^{\text {"18 }}$ Der

17 Der Maler und Brandes pflegten einen jahrelangen Briefaustausch (vgl. CHRISTENSEN 2010:122-149).

18 (о. A.) (1992): Der Zeit ihre Kunst. Über Max Klinger. In: Kunstpresse. Kunstforum Wien 1:29-33: http://www.zaunschirm.de/klinger.html (01.11.2011). 
Dritte in dieser Szene ist der über den Verlust der Gemahlin verzweifelte Amor. Auf dem zweiten, einem noch umstritteneren Bild Klingers vollzieht sich abermals eine Art ,Vermählung' der Antike mit dem Christentum. Hier geschieht dies aber nicht durch Einbeziehung mythologischer oder biblischer Figuren, sondern durch das Motiv der Nacktheit: Es sind fünf nackte Männerfiguren zu sehen, ${ }^{19} \mathrm{zu}$ denen die bekleideten Figuren in der linken Bildmitte durch ihre Statik und Gekünsteltheit im Kontrast stehen. Diese Opposition thematisiert Klinger in seiner theoretischen Schrift Malerei und Zeichnung von 1891 (vgl. KLINGER 1987), ${ }^{20}$ wo er das bekleidete Christentum gegenüber der unbekleideten Antike beklagt. „Die Forderung des Nackten“ (KLINGER 1987:54) legitimiert er - unter Berufung auf die antike Kunst - durch das Studium des menschlichen Körpers, insbesondere der im Becken zentrierten Bewegung, der für die Komposition des Bildes primär - der „Kern- und Mittelpunkt aller Kunst" (KLINGER 1987:55) - sei und nicht verdeckt werden soll: ${ }^{21}$ „Das Studium und die Darstellung des Nackten sind das A und das O jeden Stils.“ (KLINGER 1987:52) In ,allen heutigen Kunstrichtungen“ sieht Klinger dagegen die Darstellung des menschlichen Körpers in den Hintergrund treten, ein bedauerlicher Zustand, dem nicht mit „falscher Antikisierung“ beizukommen ist. „Denn nur wer ganz frei vor dem menschlichen Körper gestanden und gearbeitet hat, kann die Höhe der Leistung anderer Stilepochen empfinden.“ (KLINGER 1987:59f.)

In Franziska nimmt Wedekind das Motiv der Nacktheit im 6., der Spiel-imSpiel-Konvention verpflichteten Bild auf und stellt es analog zu Klinger in den Dienst einer Kunsttradition. Zum Anlass der Wiedereröffnung des Hoftheaters soll das Festspiel des Herzogs - ,ein harmloser Scherz, weiter nichts“ (WEDEKIND 1990a:688), aber dennoch sein „rückhaltloses Bekenntnis“ (WEDEKIND 1990a: 689) - aufgeführt werden. Letzteres bezieht sich auf den Frauenkörper, der

19 Die Christus-Figur ist nicht frontal, sondern perspektivisch verkürzt dargestellt und an einem schiefen Kreuz stehend. Dies vergegenwärtigt aber erst die Darstellung der Schächer, insbesondere der gewendete Kopf des einen. Der andere - im Vordergrund des Bildes - ist von dessen Rand abgeschnitten und zu dem Gekreuzigten in der Bildmitte hin gerichtet. Zuerst als Privatdruck erschienen.

21 Vgl. auch: „Nur am frei gegebenen Körper entwickelt sich ein gesunder Kunstsinn. Wollen wir diesen und einen gesunden Stil, so müssen wir gesunden Sinn genug haben, das Nackte nicht nur zu ertragen, sondern es sehen und schätzen zu lernen.“ (KLINGER 1987:59) 
nicht dem Blick entzogen werden sollte: „Als wäre es etwa normal, selbstverständlich, folgerichtig, dass ebenmäßig geschaffene Frauen ihren Wuchs nicht zeigen dürfen!“ (WEDEKIND 1990a:690) Diese liberale Ansicht eines ,Frauengenießers' erstreckt sich auf die Kunst und die Religion als Ausdruck des Zustands der gegenwärtigen Kultur. Der Herzog beklagt, dass der Tanz - im Gegensatz zu Musik, Plastik und Malerei, die ,als Ausdrucksmittel der Verehrung allgemein im Gebrauch“ (WEDEKIND 1990a:689) seien - bei der Andacht keine Verwendung finde. In seinem Festspiel will er sich auch die Freiheit nehmen, die Nacktheit zur Anschauung zu bringen. In die mittelalterliche $\mathrm{Ku}$ lisse des ,Einakters“ werden antike Elemente integriert: er spielt in ,hügeliger Waldlandschaft auf der Insel Rhodus" (WEDEKIND 1990a:702) und ist eine dramatische Umsetzung des Renaissancegemäldes von Tizian - Himmlische und irdische Liebe (um 1515). ${ }^{22}$ Tizian lässt hier antike und christliche Motive einfließen, was Wedekind zur Profilierung des eigenen Stücks einsetzt. Die Kulisse ist folgende:

Hügelige Waldlandschaft auf der Insel Rhodus. Im Hintergrund rechts ein Kirchturm, links auf einer Anhöhe ein Schloß, vor dem zwei Kaninchen grasen. In der Mitte der Bühne befindet sich ein breites, marmornes Brunnenbecken, dessen Außenseite mit Skulpturen geschmückt ist. Die Skulpturen zeigen spielende Kinder, die einen Triumphzug und eine Stäupung vor dem Schandpfahl darstellen (WEDEKIND 1990a:702).

Das Arrangement des Ausgangsbildes steht zwar im Zeichen des Idyllischen, wird aber bereits vom Prolog konterkariert, der auf eine verfremdende Störung vorbereitet. ${ }^{23}$ Das darauffolgende Ineinanderfließen von Zitaten folgt weitgehend demselben Prinzip. Das eigentliche Festspiel geht auf die Legende von

22 Wedekind kannte das Gemälde aus der Münchner Schack-Galerie. Eine Anspielung auf den Titel des Gemäldes taucht bereits in Fritz Schwiegerling (1892) auf: Cölestin zu Katharina: „Ich lasse viel zurück... Meine irdische und meine himmlische Liebe..." (WEDEKIND 2000:423).

23 Im Prolog zum Spiel im Spiel wendet sich Veit Kunz ad spectatores und informiert über eine Intrige, die die Aufführung stören sollte, wobei er sich auf den italienischen Renaissanceschriftsteller Pietro Aretino beruft: „Pietro Aretino war ein Spötter, / Und trotzdem hat ihn Tizian gemalt. / Auch ich bin meinem Vaterland kein Retter. / Ich kämpfe nur, solang man mich bezahlt." Wedekind (1990a:702). Aretino war mit Tizian befreundet, der Maler hatte auch zwei Porträts von ihm angefertigt (um 1545). $\mathrm{Zu}$ Wedekinds Zeiten waren Aretinos Gespräche in deutscher Übersetzung verfügbar (vgl. AUSTERMÜHL / KIESER / VINÇON 2009:1064f.). 
Georg, dem Drachentöter zurück: Der zweiköpfige, in den ,heiligen Hain“ eingedrungene Drache bedroht Franziska - hier die Verkörperung der Wahrheit, und „nur mit einem Schleier um die Hüften bekleidete“ Gislind (WEDEKIND 1990a:704) - das Symbol der ,heiligen Nacktheit" (vgl. WEDEKIND 1990a: 706). Die beiden Frauen entsprechen so dem dualistischen Prinzip auf dem zitierten Gemälde Tizians (vgl. AUSTERMÜHL / KIESER / VINÇON 2009:1038). Bellend und grunzend tritt das mit einem Hunde- und einem Schweinekopf ausgestattete Ungetüm ${ }^{24}$ als quasi-doppelte Moralinstanz auf, die „Unzucht, Laster, Ketzerei“ sowie „Völlerei“ (WEDEKIND 1990a:705) bekämpfen will. Der Herzog bezwingt als Ritter Georg den Drachen, nachdem er ein Lob auf die Nacktheit ausgesprochen hat: „Der Nacktheit denk ich strengstens einzuschärfen: / Du sollst deine Nacktheit nicht vor die Säue werfen! [...] Denn wer die Nacktheit nicht sehen kann, / Der kann auch die Wahrheit nicht hören.“ (WEDEKIND 1990a:708) Wie zuvor angekündigt tritt eine „Störung“ (WEDEKIND 1990a:708) des Festspiels ein: der Polizeipräsident verletzt die Konvention, indem er ohne Kostüm und nicht in der vorgesehenen Rolle als „Ordensmeister des Johanniterordens auf Rhodus“ (WEDEKIND 1990a:708), sondern als Ordnungshüter und Beschützer der ,öffentlichen Meinung' auftritt:

Der normale Staatsbürger kann nun einmal die Wahrheit nicht hören und die Nacktheit nicht sehen, ohne außer Rand und Band zu geraten, ohne gemeingefährlich zu werden. [...] Meine Aufgabe ist es, die öffentliche Meinung zu schützen. Auch in einem monarchischen Staate kann sich eine Regierung nicht gegen die öffentliche Meinung behaupten. (WEDEKIND 1990a:709)

Wedekinds Umdeutung des Motivs geht auf Schillers Romanze Der Kampf mit dem Drachen (1799) zurück, worin der Drachentöter als Gefährdung der Ordnung betrachtet und dementsprechend von der Kirchenobrigkeit statt wie zu erwarten Lob - Tadel erntet (vgl. AUSTERMÜHL / KIESER / VINÇON 2009:1065f; auch AUSTERMÜHL 2003:14). Mit der dreifachen Anrufung der ,öffentlichen Meinung' spielt der Text zudem auf Jacques Offenbachs Operette Orpheus in der Unterwelt (1858) an. ${ }^{25}$ In Offenbachs Persiflage des Stoffes verhindert die

24 Das Fabelwesen wird nicht illusionistisch, sondern in Masken dargestellt, die die Gesichter frei lassen und ,die Deutlichkeit der Sprache durch nichts beeinträchtig[en]“" (WEDEKIND 1990a:705).

Im Simplizissimus v. 13.12.1898 wurde Wedekinds Gedicht An die öffentliche Meinung unter dem Pseudonym Kaspar Hauser erstveröffentlicht. Es ist ein politisches Gedicht, in dem der Autor u. a. die Übergriffe der Zensur und generell die Ignoranz der Machthaber im wilhelminischen Staat thematisiert. Wedekind greift auf die mythologische Figur der klagenden Prophetin Kassandra zurück, deren 
,Öffentliche Meinung ' - die drittwichtigste Person nach Orpheus und Eurydike - die Inanspruchnahme von Freiheiten, die ihr als zu liberal erscheinen. ${ }^{26} \mathrm{Wie}$ Offenbach nutzt Wedekind die Gelegenheit, seinen Zeitgenossen einen Spiegel vorzusetzen, den ,normalen Staatsbürger ${ }^{6}$ zu denunzieren. ${ }^{27}$ Beinahe wörtlich zitiert Veit Kunz' Nebenbuhler Breitenbach den Polizeipräsidenten, wenn er beim Anblick der ,in der Unterwelt so leicht geschürzt herumstrolchenden“ Helena ,aus den Fugen“ geht, ,außer Rand und Band“ gerät und ,gemeingefährlich“ wird. (WEDEKIND 1990a:715) Das an der Störung gescheiterte Festspiel des Herzogs wiederholt, in Anspielung auf die Renaissance, Klingers Postulat der Nacktheit in der Kunst. Die Hommage ist zugleich ein kritischer Kommentar zur die Nacktheit ausschließenden gegenwärtigen Kunst und Kultur. ${ }^{28}$ Damit bezieht auch Wedekind die Position eines Sensualisten, der - in Anlehnung an die Antike und Renaissance - dem Körper eine zentrale Rolle auf der Bühne einräumt. Dass das Spiel im Spiel poetologischen Charakter und eine Schlüsselfunktion für das Stück hat, lässt sich dem an Tizians Gemälde orientierten Umschlagbild des Erstdrucks von 1912 entnehmen. Franziska, „einen Blumenkranz im Haar“, stellte „die himmlische, leidvolle Liebe“, Gislind „die fröhliche, irdische“ dar (AUSTERmüHL / KIESER / VINÇON 2009:1052f.). ${ }^{29}$ Die Stilisierung Franziskas zum Inbegriff der Wahrheit und einer reinen Liebe im Festspiel wiederholt Wedekind am Schluss des Stücks.

Weissagungen nicht erhört werden (vgl. AUSTERmüHL / KiESER / VinÇON 2007:503f. u. 926f.).

26 So kann sich Orpheus nicht ohne weiteres von Eurydike trennen, sondern wird immer wieder von der Öffentlichen Meinung, die ihn zunächst auf den Olymp, dann in die Unterwelt begleitet, dazu gebracht, sich um sie zu bemühen bzw. sie zurückzufordern.

27 Dieses Postulat wird im folgenden (8.) Bild Veit Kunz in den Mund gelegt, als sein eigenes Stück probiert wird: „Mir kam es natürlich nur darauf an, bevor die Gottheit über Satan triumphiert, das stumpfsinnig spießbürgerliche Alltagstreiben zu schildern, in der sich die Bewohner der Hölle seit Jahrhunderten mit ihren Qualen zurechtgefunden haben.“ (WEDEKIND 1990a:717). „Mit dem Drachen verspottete der Verfasser das Publikum.“ (KuTSCHER 1931:128)

28 In Reaktion auf die „Störung“ fällt der Herzog selbst aus der Rolle: „Aber in Versen, mein Lieber! In Versen! Sie sollen mich in Versen verhaften! Er quittiert die Panne dann auch resigniert: „Das ist moderne Schauspielkunst.“" (WEDEKIND 1990a:709).

29 Der Hundekopf sollte auf dem Umschlag die Wahrheit, der Schweinekopf die Nacktheit bedrohen (KuTSCHER 1931:127). (Vgl. AUSTERMÜHL / KIESER / VINÇON 2009:1052f.). 
Der Maler Karl Almer (Anagramm zu Maler) fertigte ein Porträt Franziskas mit ihrem inzwischen geborenen Sohn an: „Das Bild zeigt Franziska in halber Figur, den nackten Veitralf auf dem Arm haltend". Ihr Gesichtsausdruck erhielt, so der Maler, durch die Sorge um den kranken Sohn „etwas Leidendes“. (WEDEKIND 1990a:740) „Unten herum“ hat Almer „den Kranz aus Rosen“ gemalt, ein Element, das er als „Erinnerung an irgendein Madonnenbild“ (WEDEKIND 1990a:740) erklärt. Die Szene sorgte für Kontroversen unter den Theaterkritikern. Die bis dahin Autonomie anstrebende Protagonistin soll nun zu einem anspruchslosen bürgerlichen Leben mit Mann und Kind zurückgefunden haben (vgl. VINÇON 1987:233). ${ }^{30}$ Diese Auslegung widerspricht allerdings dem im Stück realisierten Figurenkonzept der Metamorphose. Die jeweiligen Bilder inszenieren beide Hauptfiguren - Franziska und Veit Kunz - in ihren Verwandlungen, so dass sie sich nicht auf eine eindeutige Zuschreibung festlegen lassen, sondern vielmehr als Synthese all ihrer Verkörperungen fungieren. In diesem Sinne kann die allegorische ,Nacktheit" bzw. ,irdische Liebe“ als ein Alter Ego Franziskas gelesen werden. Eine solche Perspektive eröffnet auch die letzte Szene, in die - durch Bildzitate - ebenfalls kontrapunktische Botschaften einfließen.

Als Prototyp für Wedekinds Titelfigur gilt Franziska zu Reventlow, Schriftstellerin und bekannte Persönlichkeit der Münchner Bohème (vgl. MARTIN 2001; AUSTERMÜHL / KIESER / VINÇON 2009:1040f.). Besonders das neunte und letzte Bild des Dramas rekurriert auf zu Reventlow. Das madonnenhafte Porträt Franziskas ist durch allseits bekannte Fotographien der Reventlow inspiriert, auf denen sie mit ihrem Sohn Rolf abgebildet ist. In den BohèmeKreisen Münchens wurde Franziska zu Reventlow auch als ,heidnische Madonna mit dem Kinde" bzw. als ,heidnische Heilige' bezeichnet und als Verkörperung des antiken Hetärentums verehrt. ${ }^{31}$ Ariane Martin zufolge handelt es sich um ein optisches Zitat: Da Fotographien um die Jahrhundertwende zu Gemälden stilisiert wurden, habe Wedekind gleich daraus ein Gemälde gemacht (vgl. MARTIN 2001:84). Der Sohn von Reventlows war - wie Franziskas Veitralf ${ }^{32}$ - ein uneheliches Kind, dessen Vater unbekannt blieb. Durch diese Folie

30 Vgl. zur Rezeption von Franziska auch AUSTERMÜHL / KIESER / VINÇON (2009:1079f.).

31 Die Bezeichnung 'Madonna mit dem Kinde' sollte Ludwig Klages, eine Zeitlang Lebenspartner der Reventlow, geprägt haben. Klages war Mitglied des „Kosmiker“Kreises um Stefan George (vgl. MARTIN 2001:84; AUSTERMÜHL / KIESER / VinÇON 2009:1040f. u.1058).

32 Der Name des Kindes vereint die Vornamen beider in Frage kommenden Väter - Veit Kunz und Ralf Breitenbach. 
gelesen wird die vermeintliche ,Bürgerlichkeit' des letzten, umstrittenen Bildes unmissverständlich relativiert. ${ }^{33}$

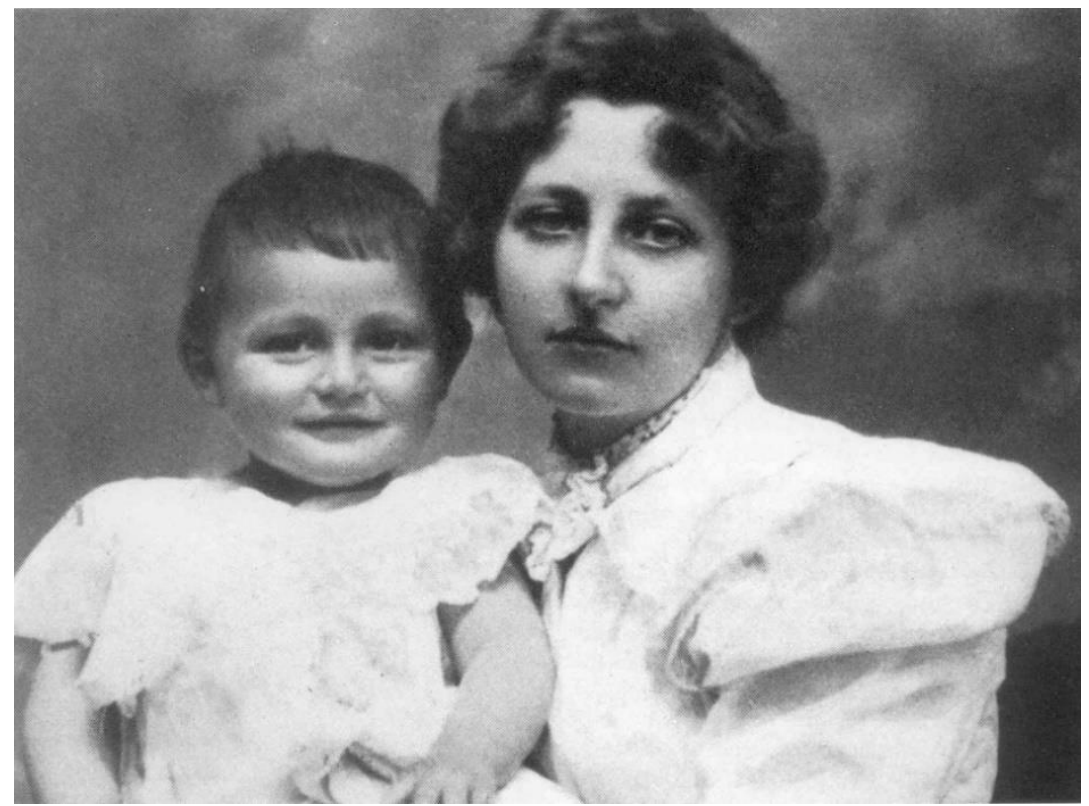

Abb.: Fanny Gräfin zu Reventlow mit Sohn Rolf, 1898 (Fotograph unbekannt) ${ }^{34}$ Das Madonnenbild steht allenfalls auf der Schwelle zwischen Sakralem und Profanem, wobei der Freigeist, der durch das Fotozitat einfließt, als eine Art ironisches Korrektiv der christlichen Tradition zu fungieren scheint. Eine ähnliche Konstellation ist bereits in Frühlings Erwachen vorzufinden, worin die sexuell emanzipierte Ilse dem Maler Landauer für die ,Heilige Maria, Mutter Gottes mit dem Christuskind“ Modell sitzt. ${ }^{35}$ Zwei Tagebucheinträge

33 Riedlinger argumentiert mit Wedekinds Anlehnung an Nietzsche: Franziska habe er als einen „weiblichen Übermenschen“ gestaltet, den ,antibürgerliche Moral“ (RIEDLINGER 2005:112) kennzeichnet.

34 https://de.wikipedia.org/wiki/Datei:Reventlow_Madonna.jpg (28.08.2017).

35 Ilse: „Ich saß den Tag bei Isidor Landauer. Er braucht mich zur heiligen Maria, Mutter Gottes, mit dem Christuskind.“(WEDEKIND 1990b:514) Ähnlich ironische Äußerung Ilses: „Fehrendorf malt mich als Säulenheilige. Ich stehe auf einem korinthischen Kapitel.“(WEDEKIND 1990b:513) 
Wedekinds von 1889 zeugen von seiner großen Empfänglichkeit für Madonnabildnisse, die aber kaum religiösen, sondern vielmehr einen sensualistischen Charakter hat. So heißt es in einem der Beiträge: „Diese Züge [Profil eines Schwans. J. F.] verleihen dem Schwan den ernsten sinnigen Ausdruck, der sich zu demjenigen des griechischen Profils verhält wie die Madonnenköpfe Andrea del Sartos zu denjenigen Raphaels.“" (vgl. HAY 1986:136) ${ }^{36}$ Das spannungsvolle Zusammenspiel von Madonnenhaftigkeit und Sinnlichkeit erkennt er auch in Tilly Newes, deren Spiel in der Aufführung seiner Büchse der Pandora in Wien 1905 ihn für sie einnimmt: „Daß das Publikum mein abscheuliches Stück ohne dein kluges und zugleich so madonnenhaftes Spiel nicht geduldig hingenommen hätte, darüber besteht für mich nicht der geringste Zweifel.“ (STRICH 1924b:143) ${ }^{37}$ Bei der Konstruktion der Franziska-Figur scheint diese Ambivalenz besonders im letzten Bild ausschlaggebend zu sein. Der Bezug zur heiligen Madonna wird nicht nur durch die Folie der Reventlow, sondern auch durch die Parallele zur Helena-Figur konterkariert. Diese wird bereits im sechsten Bild des Wedekind'schen Stücks hergestellt. Der Dramatiker bedient sich hier abermals des Spiel-im-Spiel-Modells. Im Mysterium des Veit Kunz tritt Franziska in der Rolle der jungen Helena von Sparta auf. Das Konzept dieser Einheit entspricht dem Konzept des gesamten Stücks: Auch hier wird die christliche Idee des Mysteriums durch eine Reihe von Zitaten überblendet und dekonstruiert, so bei Anleihen aus Dantes Divina Comedia. Helena gehört bei Dante den Höllenbewohnern an, sie befindet sich unter ,Wollüstigen', die im zweiten Höllenkreis büßen. Im neunten Bild ist die Anspielung auf diese Helena-Figur zwar indirekt, aber unübersehbar und zwar insbesondere durch Elemente der Faust-Stoff-Bearbeitungen. Zum einen taucht im älteren Faustbuch das Motiv eines Helena-Konterfeis auf (vgl. HEINE

36 Wedekinds Notiz v. 2.09.1889, München. Andrea del Sarto (1486-1530), bedeutender Kolorist der Florentinischen Renaissance. Im zweiten Eintrag berichtet Wedekind von einem seiner Besuche in der Münchner Schack-Galerie: ,[G]ehe in die Galerie Schack, wo mich besonders die geistreichen Kompositionen von Genelli interessieren. [...] Lange Zeit verbringe ich vor der Madonna von Andrea del Sarto. Das ist wirklich ein Mädchen, dem der heilige Geist auf der Stirne leuchtete. Das wäre mein Geschmack. Der kluge Ernst in diesen Zügen wäre mir zehntausendmal lieber als die Liebe in denjenigen der Sixtinischen. So wie diese heilige Jungfrau denke ich mir Anna Launhardt. Wäre ich Andrea del Sarto, ich hätte das Mädchen Medizin studieren lassen." (HAY 1986:138), Eintrag v. 03.09.1889.

Wedekind an Tilly Newes, 4.06.1905, München. 
1962:41). In der Historia von Dr. Johann Fausten sowie in Goethes Faust II wird Helena - der Inbegriff der Schönheit und Kraft, der klassischen Antike und des romantischen Mittelalters - fürsorgliche Mutter eines Sohnes von Faust (vgl. AuSTEMÜHL 2003:8). ${ }^{38}$ Der Maler Almer stattet das Porträt Franziskas allerdings mit einem ambivalenten Detail aus: einem Kranz aus Rosen zu Füßen des Modells. Almer kann sich an die Inspirationsquelle nicht genau erinnern und weist in diesem Zusammenhang auf „irgendein Madonnenbild“ (WEDEKIND 1990a:740) hin. Die Rosen werden in der christlichen Symbolik der heiligen Maria als Symbol der Liebe, Anbetung und Hingabe (vgl. BUTZER / JАСОВ 2008:301-304) $)^{39}$ sowie der Unschuld ${ }^{40}$ zugeordnet. Das Rosensymbol steht aber auch für erotische Liebe und die erotisch konnotierten Körperteile. Diese ,heidnische" Deutung bestätigt bei Wedekind die Komposition der Blumen zum Kranz. ${ }^{41}$ Darstellungen der Madonna mit dem Kind sind in großer Zahl vorhanden, besonders ergiebig war in dieser Hinsicht die Renaissance. Dies gilt aber nicht für Darstellungen der Madonna mit einem Rosenkranz zu ihren Füßen. Auf dem Gemälde Helena von Troja (1898) der britischen Malerin Evelyn de Morgan (Pickering) (1855-1919) wird die Titelfigur stehend gezeigt. Sie hält einen altsilbernen Spiegel in der Hand und besieht sich darin, während die dem Betrachter zugewandte Rückseite des Spiegels die Gestalt Helenas in derselben Haltung wie auf dem Bild wiederholt. Evelyn De Morgan schuf in dem sich gerade verbreitenden Stil der PräraffaelitInnen, auch wenn sie nicht zu deren HauptvertreterInnen gezählt wird. Die Präraffaeliten wurden in Deutschland durch Hugo von Hofmannsthals Essays aus den 1890er Jahren rezipiert, ${ }^{42}$ die Wedekind bekannt gewesen sein dürften, denn beide waren Mit-

Zum Motiv der Mater Gloriosa am Schluss von Faust II vgl. AustermüHL 2003:4. Vgl. auch http://kath.de/kurs/symbole/rose.php (29.10.2011)

Alle drei Fassungen des Stücks enthalten den Verweis auf den Bildtypus der ,Madonna im Rosenhag‘ vgl. AustermüHL / KIESER / VinÇON 2009. Begründet wurde dieser Typus von Stefan Lochner (1448) und weiterentwickelt u. a. von Martin Schongauer (Altarbild für die Dominikanerkirche in Colmar v. 1470) und Sandro Botticelli (1470).

41 Zum (Blumen)Kranz als Symbol für die Vulva, zudem als Symbol der Ehrung in christlichen wie heidnischen Riten (vgl. BUTZER / JACOB 2008:52f.).

Hofmannsthal hat den Präraffaeliten (Kunst des mittleren Viktorianischen Zeitalters, etwa 1848-1875) vier Essays gewidmet: Algernon Charles Swinburne, 1893; Über moderne englische Malerei, 1894; Walter Pater, 1894; Englischer Stil, 1896 (vgl. HöNNIGHAUSEN 1992/2000:363-372). 
arbeiter des Münchner Simplizissimus. Denkbar ist auch Wedekinds Kenntnis des der englischen Kultur und Ästhetik gewidmeten Buches Die Mystik, die Künstler und das Leben (1900) des Kulturphilosophen, Essayisten und Erzählers Rudolf Kassner (vgl. KASSNER (1992/2000), der um 1900 eine Zeit lang in München lebte und zum Umkreis der Münchner Bohème und damit zum Bekanntenkreis Wedekinds gehörte. Ob Wedekind aber das Gemälde Helena von Troja kannte, ist nicht mit Sicherheit festzustellen, zumal sich De Morgan nicht besonders um ihre Popularität bemühte, auch wenn sie einige Ausstellungen in England hatte. ${ }^{43} \mathrm{Ihr}$ Name taucht zudem in der Münchner Zeitschrift Die Kunst. Monatsheft für freie und angewandte Kunst 9 aus dem Jahr 1904 auf. (o. A. 1904:88) Vielleicht hatte Wedekind das Bild der englischen Malerin gesehen, ohne sich an die Quelle erinnern zu können, wie Almer es erklärt? (WEDEKIND 1990a:740) Die Affinität zu Franziska-Figur erschöpft sich nicht im Rosenkranzmotiv. Bemerkenswert ist zudem das Motiv des Spiegels, das in Wedekinds Stück dramaturgisch wiederholt wird, als Franziska „Veitralf in den Armen haltend" (WEDEKIND 1990a:740) das eigene Porträt betrachtet. Nicht zuletzt ist auf die ideelle Verwandtschaft zwischen dem Stück und der Malerei der Präraffaeliten hinzuweisen, wie sie sich auch im Helena-Bild der englischen Malerin manifestiert. Die als die bedeutendste Ausprägung der viktorianischen Malerei geltende Kunstrichtung der Präraffaeliten verband Historismus - die Rückbesinnung auf Stil und Technik der alten Meister - mit dem modernen, dokumentarischen Realismus des 19. Jahrhunderts, der Epoche der Fotographie und der Empirie (vgl. BARRINGER 1998:8-10). De Morgans Gemälde aus den 1890er Jahren sind zum großen Teil von Botticelli inspiriert.

Die Künstlerin verband mythologische und biblische Motive, ihre Frauendarstellungen assimilieren Christliches und Heidnisches. ${ }^{44}$ Helena stellt ein sensualistisches, verführerisches Frauenbild dar. Durch Rosen- und Tauben-

43 Ausstellung im Leighton House, London (1902f.); Ausstellung Anglo-Florentine Portraits in der Bruton Gallery, London (1906); Ausstellung von 25 Gemälden in der Wolverhampton Art Gallery (1907) sowie Red Cross Benefit Exhibition im Edith Grove Studio, London (1916) (vgl. GoRDON 1996).

Z.B. in der Driade (1884f.), wo die heidnische Nymphe zum Symbol der Erlösung (redemption) und der Wiedergeburt wird. Vgl. LAWTON SMITH, Elise (1997f.): The Art of Evelyn De Morgan. In: Woman's Art Journal 18, Nr. 2:3-10. Online verfügbar: http://www.jstor.org/stable/1358544 (17.10.2011). 
Attributierung ${ }^{45}$ wird eine symbolische Beziehung zu Aphrodite ${ }^{46}$ und zur christlichen Madonna ${ }^{47}$ hergestellt. Im bürgerlichen Ambiente des neunten Bildes wird Franziska durch das Gemälde De Morgans mit der heidnischen Helena als Allegorie der Kunst und Madonna überblendet, womit die Idee einer Verbindung von Antike und Christentum bzw. Eros und Religion ${ }^{48}$ in der Ordnung der Kunst und nur dort umgesetzt wird. Denn die Handlung vermittelt eine in dieser Hinsicht negative bzw. in die weite Zukunft projizierte Botschaft: Erst in zweitausend Jahren könne die Heidin Helena / Franziska „zum Licht geleitet werden“ (WEDEKIND 1990a:723): Veit Kunz als Christus / Orpheus: „Ich muss die Macht für größeres mir bewahren. / Doch steigt herab und hebt zum Himmel dich / Vielleicht ein andrer in zweitausend Jahren.“ (WEDEKIND 1990a:723) Das ist auch die als denkbar hingestellte Perspektive, bis „die Kirche wieder so klug sein [wird], die Nacktheit heilig zu sprechen!“ (WEDEKIND 1990a:711) Das ambivalente Franziska-Porträt besiegelt also lediglich die Idee einer Synthese antiker und christlicher Kultur, die auch anderen optischen Zitaten zugrunde liegt. Mit Franziska scheint Wedekind eine Antwort auf Thomas Manns Novelle Gladius Dei geben zu wollen, in der der Autor ein parodistisches Bild Wedekinds als Moralisten zeichnet. Die Novelle spielt im zeitgenössischen München, wo die Kunst im Zug der missbrauchten Vervielfältigungsrechte und -techniken längst ihre Aura verloren hat. Die Meister des Florentiner Quattrocento - Donatello und Minno da Fiesole - werden in den „kleinsten und billigsten Läden“ (vgl. MANN 1993:193) als Kopien verkauft. Den Höhepunkt des Textes bildet die Begegnung einer mönchshaften Figur namens Hieronymus mit Blütenzweig -

45 De Morgans Helena ist durch weiße Tauben attributiert. Dieses Motiv taucht auch bei Wedekind im 6. Bild, in der Begegnung Helenas mit dem Erlöser Veit Kunz auf: „Der Schwan ein Greuel, ein Idol die Taube!“ (WEDEKIND 1990a:723). Helena wurde ,vom Schwan gezeugt, wie er [die Gottheit] von der Taube“ (Kutscher 1931:128). Zeus soll sich Leda in Gestalt eines Schwans genähert haben, eine Ver-bindung, aus der Helena hervorging. Der Schwan gilt als Symbol der Schönheit und Reinheit, ein Attribut Aphrodites (vgl. AustermüHL / KIESER / VINÇON 2009:1067).

Vgl. Hartmann, P. W. (o.J.): Das große Kunstlexikon: http://www.beyars.com/ kunstlexikon/lexikon_532.html (14.10.2011).

47 In der christlichen Symbolik sind Tauben ein Zeichen des Geistes (vgl. BuTZER / JАСОВ 2008:301-304; vgl. auch http://kath.de/kurs/symbole/taube.php (29.10.2011)).

Zu Wedekinds Religionsbegriff vgl. SCHRÖDER-ZEBRALLA (1985:141f.). 
dem Hersteller fotographischer Reproduktionen in dessen Kunsthandlung am Odeonsplatz. Hieronymus - „ein seltsamer Heiliger, ein Savonarola des Fin de Siècle, ein Asket und religiöser Eiferer" fordert den Besitzer dazu auf, eine nach einem Mariengemälde angefertigte Fotographie aus dem Schaufenster zu entfernen. Es handelt sich um „,de[n] Typus einer Maria Lactans, die Madonna also, wie sie ihr Kind stillt, doch in diesem Fall kommt sie ihrer Mutterschaft etwas zu barbusig nach und lässt noch von ungefähr Reminiszenzen an Stucks Sünde aufkommen.“ (METZGER 2009:190) ${ }^{49}$ Hieronymus wird von einem gewissen Krauthuber, dem Sohn des Volkes, aus dem Laden hinausgeworfen, so dass ihm allein die Hoffnung auf ,Gladius Dei', das Schwert Gottes bleibt, das kommen möge. Der private Zensor heißt bezeichnenderweise genauso wie Frank Wedekind im Pseudonym, unter dem er im Simplizissimus seine Gedichte veröffentlichte. Es ist hochironisch, wenn der rebellische Geist Wedekind, dem die Zensurbehörden stets das Leben verleideten, von Thomas Mann zu einem Moralverkünder stilisiert wird. Mit der Gestalt der scheinbar domestizierten Franziska scheint ,Hieronymus“ eine provokante Antwort auf die Herausforderung Thomas Manns bieten zu wollen. Frank Wedekinds Sensibilität für die bildende Kunst, die Einsicht in sein ,Denken in Bildern', in die Visualisierungstendenz, die seinen Texten zugrunde liegt, ermöglicht einen neuen Zugang zum Schaffen des Dramatikers. Mit einer stark ausgeprägten Szenenchoreographie und zahlreichen Kunstzitaten stellt das späte Stück Franziska ein paradigmatisches Beispiel hierfür dar. Das reichhaltige Bildarchiv, angefangen mit dem Faust-Stoff über Mignon-, Eva-, Maria Magdalena- und Helena-Figuren, über Gemälde Tizians, Max Klingers, der Kubisten und Präraffaeliten begründet die Position Wedekinds als Kenner des Kunstdiskurses seiner Zeit und als Sensualisten, der dem Optischen eine zentrale Rolle einräumt. ${ }^{50}$ Dabei sind die Traditionen und Stoffe, auf die der Autor rekurriert, durchaus nicht beliebig, sondern in ein übergeordnetes Koordinatensystem eingebunden. Wedekind bekennt sich zu einer Art synthetischer Anthropologie, die sich im Konzept der Figuren als Konstrukte am plakativsten manifestiert und nach der Antike und Renaissance ein Korrektiv zur christlichen Tradition darstellt und den christlich tradierten Dualismus von Geist und Körper relativiert.

\footnotetext{
$49 \quad$ Franz von Stucks (1863-1928) Sünde v. 1893.

50 Wedekind sei ein Sensualist und Nachfahre Heinrich Heines (vgl. IRMER 1975:13).
} 


\section{Literatur}

AdORNO, THEODOR W. (1974): Über den Nachlass Frank Wedekinds (1932). In: TIEDEMANN RolF unter Mitwirkung von AdORnO, GRETEL / BuCK-MORSS, SuSAN / SCHUlTZ, KLAUS (eds.): Theodor W. Adorno. Gesammelte Schriften. 20 Bde. in 23 Teilbänden, Bd. 11. Frankfurt a. M., 627-633.

AUSTERMÜHL, ELKE (1989): Eine Lenzburger Jugendfreundschaft. Der Briefwechsel zwischen Frank Wedekind und Minna von Greyerz. In: AUSTERMÜHL, ELKE / KESSLER, Alfred / VinçON, HARTMUT (eds.): Kein Funke mehr, kein Stern aus früh'rer Welt. Frank Wedekind. Texte, Interviews, Studien. (Pharus 1) Darmstadt, 343-420.

AUSTERMÜHL, ElKe (2003): Wedekinds „Franziska“ - ein weiblicher Faust. In: HÄrTER, ANDrEAs / KunZ, Edith ANna / WeIdMAnN, HeInER (eds.): Dazwischen. Zum transitorischen Denken in Literatur- und Kulturwissenschaft. Göttingen, 79-100.

AustermüHL, ElKe / VinçOn, HARTMut / Kieser, Rolf et al. (eds.) (2007): Frank Wedekind. Historisch-kritische Studienausgabe der Werke. Bd. 1 in 2 Teilbänden. Darmstadt.

- (2009): Frank Wedekind. Historisch-kritische Studienausgabe der Werke. Bd. 7 in 2 Teilbänden. Darmstadt.

- (2000): Frank Wedekind. Historisch-kritische Studienausgabe der Werke. Bd. 2. Darmstadt.

BARringer, Tim (1998): Die Präraffaeliten. Wie sie malten, wie sie dachten, wie sie lebten. Köln.

BUTZER, GÜNTER / JACOB, JOACHIM (eds.) (2008): Metzler Lexikon literarischer Symbole. Stuttgart.

Christensen, Charlotte (2010): Max Klinger und Georg Brandes. In: HütTel, RICHARD / SCHMIDT, HANS-WERNER (eds.): Max Klinger, der moderne Künstler schlechthin. Berlin / München, 122-149.

DREISEITEL, SIEGRID (2000): „Ich mache natürlich lebhaft Propaganda für ihn. “ Würzburg.

FRY, EdWARD (1966): Der Kubismus. Schauberg, Köln.

Gleizes, Albert (1928): Kubismus. Mainz.

Gordon, CATHERINe (ed.) (1996): Evelyn De Morgan Oil Paintings: http://www.mezzomondo.com/arts/mm/preraphaelites/demorgan/evelyn_de_morgan.html. (5.11.2011)

HAy, GeRHARD (ed.) (1986): Frank Wedekind. Die Tagebücher. Ein erotisches Leben. Frankfurt a. M.

HeIMBÖCKEL, DIETER (1996): Walter Rathenau und die Literatur seiner Zeit. Studien zu Werk und Wirkung. Würzburg, 142-144.

HeIne, HeInRICH (1962): Der Doktor Faust. Erläuterungen. To Lumley, Esquire, Director of the Theater of Her Majesty the Queen. In: KAUFMANn, HANS (ed.): Heinrich Heine. Werke und Briefe in 10 Bden, Bd. 7. Berlin, 28-53. 
IRMER, HANS-JOCHEN (1975): Der Theaterdichter Frank Wedekind. Werk und Wirkung. Berlin.

KAFITZ, DiETER (2001): Die Kunstzitate in Frank Wedekinds „Frühlings Erwachen “: Zur Hänschen Rilow-Szene. In: DreISEITEL, SIGRID / VInÇON, HARTMUT (eds.): Kontinuität Diskontinuität. Diskurs zu Frank Wedekinds literarischer Produktion (1903-1918). Würzburg, 263-282.

- (2006): Moderne Tendenzen in den Dramen Frank Wedekinds. In: DEsCOURVIÈRES, Benedikt / Marx, Peter W. / RÄtting, Ralf (eds.): Mein Drama findet nicht mehr statt. Deutschsprachige Theater-Texte im 20. Jahrhundert. Frankfurt a. M., 21-40.

KASSNER, Rudolf (1900): Die Mystik, die Künstler und das Leben. In: HönNIGHAUSEN, Gisela (1992/2000) (ed.): Die Präraffaeliten. Dichtung, Malerei, Ästhetik, Rezeption. Stuttgart, 373-379.

KLINGER, MAX (1987): Malerei und Zeichnung. Tagebuchaufzeichnungen. Briefe. Leipzig. KuTsCher, Artur (1931): Frank Wedekind. Sein Leben und seine Werke. In 3 Bdn. Bd. 3. München.

- (1964): Wedekind. Leben und Werk. München.

Mann, Thomas (1993): Gladius Dei. In: Thomas Mann: Sämtliche Erzählungen in zwei Bdn. Bd. 1.Frankfurt a. M., 192-209.

MARTIN, ARIANE (2001): Spiel mit Konventionen. Goethes „Faust“ und Franziska Gräfin zu Reventlow in Frank Wedekinds ,modernem Mysterium ',Franziska“. In: DreISEITEL, Sigrid / VinÇOn, HaRTMut (eds.): Kontinuität - Diskontinuität. Diskurs zu Frank Wedekinds literarischer Produktion (1903-1918). Würzburg, 75-96.

MetzGer, RAIner (2009): München. Die große Zeit um 1900. Kunst und Kultur 1890-1920. Wien.

REINHART, ERNST (1905): Von neuzeitlicher Malkunst. Zur Kritik der Moderne. In: Die Zukunft 53:11-25.

RIEDLINGER, STEFAN (2005): Aneignungen. Frank Wedekinds Nietzsche-Rezeption. Marburg. SCHRÖDER-ZEBRALLA, JOSEPHINE (1985): Frank Wedekinds religiöser Sensualismus. „Die Vereinigung von Kirche und Freudenhaus? “. Frankfurt a. M.

SteIn, LeO (1947): Appreciation. Painting, Poetry and Prose. New York.

STRICH, FriTz (ed.) (1924a): Frank Wedekind. Gesammelte Briefe. Bd 1. München.

- (1924b): Frank Wedekind. Gesammelte Briefe. Bd 2. München.

TRAPP, WilhElM: Bayerische Träumerei. Der Zeit-Museumsführer (30): Die Sammlung Schack in München. In: Die Zeit 49 v. 26.11.2009:65.

VINÇON, HARTMUT (1987): Frank Wedekind. Stuttgart.

WEDEKIND, FRANK (1990a): Franziska. In: Weidl, Erhard (ed.): Frank Wedekind. Werke in 2 Bdn., Bd. 2. München, 639-742.

- (1990b): Frühlings Erwachen. In: Weidl, Erhard (ed.): Frank Wedekind. Werke in 2 $B d n$, Bd. 1. München, 473-548. 